\title{
Drug-Resistant Tuberculosis and Lines of Mycobacterium Tuberculosis Circulating in Kinshasa
}

\author{
Marie José Kabedi Bajani ${ }^{1 *}$, Jean Marie Kayembe Tumba ${ }^{2}$, Patrick Kayembe Kalambay ${ }^{4}$, Taba Kalulu ${ }^{3}$, Paulin \\ Mbaya Kalumba $^{1}$ and Jean Jacques Muyembe Tamfum ${ }^{1}$ \\ ${ }^{1}$ Department of Medical Biology, University of Kinshasa, Democratic Republic of the Congo \\ ${ }^{2}$ Department of Medicine Internal, University of Kinshasa, Democratic Republic of the Congo \\ ${ }^{3}$ Department of Chemistry Organic, University of Kinshasa, Democratic Republic of the Congo \\ ${ }^{4}$ Department of Public Health, Technical Medical Institute of Kinshasa, Democratic Republic of the Congo
}

*Corresponding author: Marie José Kabedi Bajani, Department of Microbiology, National Institute for Biomedical Research (INRB), University of Kinshasa, Democratic Republic of the Congo Email: bedye2001@yahoo.fr

To Cite This Article: Marie José Kabedi Bajan, Drug-Resistant Tuberculosis and Lines of Mycobacterium Tuberculosis Circulating in Kinshasa. Am J Biomed Sci \& Res. 2019 - 6(5). AJBSR.MS.ID.001065. DOI: 10.34297/AJBSR.2019.06.001065.

Received: 眥 December 06, 2019; Published: 制 December 12, 2019

\begin{abstract}
Drug-Resistant Tuberculosis (DR-TB) is a public health problem in the Democratic Republic of Congo (DRC). However, knowledge of Mycobacterium tuberculosis genotypes is very important for determining the different lines involved in resistance and allowing better control of the disease.
\end{abstract}

Aim: Determine the frequency of drug-resistant strains and the different lines circulating in the City.

Material and methods: The descriptive survey covers all the family contacts of MDR-TB TB patients for whom a positive sputum culture on LJ medium was obtained in the Mycobacteria Laboratory of the University of Kinshasa between 2011 and 2017. The demographic and Epidemiological data concerning patients were collected via a registry notebook. The strains were identified by standard tests and the antibiogram was performed to obtain a chemosensitivity profile. The strains were transferred to IMT Antwerp for the genotyping test (Spoligotyping).

Results: Of 240 samples from household contacts of MDR-TB patients, 29 isolates were identified (12.1\%). Twenty-three (79\%) were strains of Mycobacterium tuberculosis and 6 (21\%) M. africanum. Overall, 16 strains of Mycobacterium tuberculosis (55.2\%) were resistant to at least one antituberculosis drug. Of the 16 drug-resistant strains, 15 (6.3\%) were MDR-TB, of which 10 (4.2\%) preXDR and 1 (0.4\%) XDR-TB. No monoresistance to rifampicin and Isoniazid was detected. The spoligotyping profile obtained shows the presence of 4 main lines namely: AML (43.7\%); West Africa (31.3\%); Haarlem (12.5\%) and Ugandal (12.5\%).

Conclusion: The results of our study show a very high primary resistance and the presence of multi and ultra-resistant strains in the community. The study also shows that there is considerable genotypic diversity among M. tuberculosis isolates in Kinshasa.

Keywords: Tuberculosis; Drug-resistant; Different lines; Mycobacterium tuberculosis; Kinshasa

\section{Introduction}

Drug-resistant TB is the form in which bacilli are resistant to TB drugs. It constitutes a public health challenge and hinders efforts to control the disease by the national control programs [15]. Early diagnosis and personalized treatment are major assets in the strategy of its eradication. Resource-poor countries, which are also the most affected, are more dramatically exposed to the spread of the disease. These countries bear $87 \%$ of morbidity/ mortality weight according to world statistics and the regions most affected are South-East Asia and Sub-Saharan Africa [1,2]. Human responsibility is very high in the development of this drug resistance, which justifies strategies centered on the speed of diagnosis and the appropriateness of treatment [1-5]. However, some published studies have revealed that several families of genotypes other than the Beijing genotype, such as the Haarlem [3] and LAM [4] families, have also shown a preponderance of drug resistance. 
Thus, knowledge of genotypic lines of circulating strains is of paramount importance, as some recognized virulent are involved in drug resistance and hinder the treatment and control of TB worldwide. Recent data published by the World Health Organization (WHO) in 2018, show 558,000 new cases of Rifampicin-Resistant Tuberculosis (RR-TB), among which $82 \%$ of people had MDR-TB and in this group, 8.5\% had UR-TB [1]. The Democratic Republic of Congo (DRC), with a population of 81 million, is cited among the countries that carry the heavy (87\%) global burden of tuberculosis and TB-HIV coinfection [1]. The WHO estimates that there are 3,400 cases of TB-drug-resistant (RR-TB and MDR-TB) according to the 2018 Global Report on Tuberculosis, including 2.2\% for new cases and $9.6 \%$ for treatment failures [1].

More recently, a survey conducted on primary resistance among people living with HIV (PLWHA), reported a proportion of $54.3 \%$ with MDR-TB around $4.3 \%$ in the city of Kinshasa [5]. This situation may be an obstacle to meeting TB elimination goals as a public health problem in the country. It is in this context that we initiated this work with suspected contacts of tuberculosis to determine the frequency of drug-resistant tuberculosis and the different genotypic lines in circulation.

\section{Material and Methods}

\section{Data collection methods}

The present descriptive study deals with contact cases of MDRTB TB patients for whom a positive culture was obtained in the mycobacteria laboratory of the University of Kinshasa between 2011 and 2017. The spoligotyping of the strains was carried out at the National Institute of Tropical Medicine in Antwerp in 2018. Demographic and epidemiological information has been extracted from the laboratory register. Two sputum samples were collected from each suspected tuberculosis contact and were sent to the bacteriology laboratory of the Faculty of Medicine for strain isolation and susceptibility testing on Lowenstein-Jensen (LJ) LJ medium using the Canetti et al. [6].

Sputum was processed by the Petroff method using $4 \%$ sodium hydroxide and the inoculum of the Ziehl positive samples were seeded directly onto two tubes containing Lowenstein-Jensen (LJ) pure medium and other tubes containing. LJ incorporated antituberculosis drugs at different concentrations namely: Isoniazid ( $0.2 \mu \mathrm{g} / \mathrm{ml})$; Rifampicin $(40 \mu \mathrm{g} / \mathrm{ml})$; Ethambutol $(2 \mu \mathrm{g} / \mathrm{ml})$; Streptomycin $(4 \mu \mathrm{g} / \mathrm{ml}) ;$ Ofloxacin $(4 \mu \mathrm{g} / \mathrm{ml})$ and Kanamycin $(30 \mu \mathrm{g} /$ $\mathrm{ml}$ ) for the direct method [6].

On the other hand, the Ziehl negative samples were inoculated only on the LJ medium and after growth of the seeds, the biochemical identification was carried out by the test of Niacin and the 2-thiophen carboxylic acid $(2 \mu \mathrm{g} / \mathrm{ml})$ [6]. Antibiosis by the indirect method of proportion was performed after isolation [6]. Genotyping of resistant Mycobacterium tuberculosis strains was performed based on the study of DR locus polymorphism (spoligotyping) according to the protocol of IMT Antwerp [7,8]. This locus is made up of short repeated identical DNA sequences called DR, separated from each other by non-repetitive and unique DNA sequences, called inter-DR or spacers $[7,8]$.

The spoligotyping genetic fingerprint is based on the study of the presence or absence of a selection of 43 spacers $[7,8]$. The DR locus is firstly amplified by PCR, and the PCR products obtained will then be hybridized on a membrane where the inter-DR $[7,8]$ is fixed. Thus, any inter-DR present in the locus previously amplified by PCR will be attached to its copy located on the membrane $[7,8]$. Fixation or non-fixation of inter-DR to their copy is revealed by chemiluminescence on photographic film $[7,8]$. The resulting spoligotype thus reflects the presence or absence of each of the 43 inter-DRs studied $[7,8]$. Strains lines were defined via the online database SITVITWEB $[7,8]$.

\section{Data analysis}

Data was analyzed on SPSS software version 20.0. Frequencies and standard deviation have been produced. The comparison of the variables was performed using the Chi-square test. The significance level was set at $5 \%$ and the $95 \%$ Confidence Interval (CI).

\section{Ethical consideration}

The study received the approval of the Ethics Committee of the School of Public Health of University of Kinshasa $\left(\mathrm{N}^{\circ} \mathrm{ESP} /\right.$ CE/081/2010).

\section{Results}

Overall 240 contacts, 111 women (46.2\%) and 129 men $(53.8 \%)$ were included in the study. The average age of contacts was 33.5 years ( $\mathrm{SD}=13.5)$. A total of 29 strains of Mycobacterium were isolated (12.1) from the 240-sputum analyzed. The identification of strains made it possible to determine two types of species, namely M. tuberculosis (79\%) and 6 M. africanum (21\%). Sixteen strains (55.2\%) out of the 29 tested were resistant to at least one anti-tuberculosis drug and $13(44.8 \%)$ were sensitive to all the molecules tested. Fifteen strains (6.3\%) were MR-TB and among them, 4.2\% preXDR [9] and 0.4\% XDR-TB [1]. No monoresistance to rifampicin and isoniazid was detected. The characterization of patients and Mycobacterium isolates according to resistance is shown in (Table 1,2).

\begin{tabular}{|c|c|c|c|c|c|c|c|}
\hline Table 1: Characterization of Patients and Corresponding Mycobacterium Tuberculosis Isolates as a Function of Resistance. \\
\hline Variables & \multicolumn{2}{|c|}{ Resistant case } & \multicolumn{2}{|c|}{ TB-MR case } & \multicolumn{2}{|c|}{ PreXDR-TB case } & \multicolumn{2}{|c|}{$(\mathrm{n}=10)$} & \multicolumn{2}{|c|}{ XDR-TB case } \\
\hline & \multicolumn{2}{|c|}{$(\mathrm{n}=16)$} & $\mathrm{n}=15)$ & $\mathrm{n}=1)$ \\
\hline & $\mathrm{n}$ & $(\%)$ & $(\%)$ & $\mathrm{n}$ & $(\%)$ & $\mathrm{n}$ & $(\%)$ \\
\hline
\end{tabular}




\begin{tabular}{|c|c|c|c|c|c|c|c|c|}
\hline Sex & & & & & & & & \\
\hline Men & 10 & 62.5 & 10 & 66.7 & 6 & 60 & 1 & 100 \\
\hline Women & 6 & 37.5 & 5 & 33.3 & 4 & 40 & 0 & 0 \\
\hline Total & 16 & 100 & 15 & 100 & 10 & 100 & 1 & 100 \\
\hline \multicolumn{9}{|l|}{ Age } \\
\hline $0-19$ & 8 & 50 & 7 & 46.7 & 2 & 20 & 0 & 0 \\
\hline $20-54$ & 5 & 31.3 & 5 & 33.3 & 7 & 70 & 1 & 100 \\
\hline 55 and over & 3 & 18.7 & 3 & 20 & 1 & 10 & 0 & 0 \\
\hline Total & 16 & 100 & 15 & 100 & 10 & 100 & 1 & 100 \\
\hline \multicolumn{9}{|l|}{ HIV status } \\
\hline Positive & 14 & 87.5 & 14 & 93.3 & 8 & 80 & 1 & 100 \\
\hline Negative & 2 & 12.5 & 1 & 6.7 & 2 & 20 & 0 & 0 \\
\hline Total & 16 & 100 & 15 & 100 & 10 & 100 & 1 & 100 \\
\hline \multicolumn{9}{|l|}{ Bacteriology } \\
\hline $\mathrm{ZN}+/$ Culture+ & 15 & 93.7 & 14 & 93.3 & 10 & 100 & 1 & 100 \\
\hline ZN-/Culture- & 1 & 6.3 & 1 & 6.7 & 0 & 0 & 0 & 0 \\
\hline Total & 16 & 100 & 15 & 100 & 10 & 100 & 1 & 100 \\
\hline \multicolumn{9}{|l|}{ Genotype } \\
\hline West Africa & 5 & 31.3 & 4 & 26.6 & 2 & 20 & 0 & 0 \\
\hline LAM & 7 & 43.7 & 7 & 46.6 & 6 & 60 & 0 & 0 \\
\hline Haarlem & 2 & 12.5 & 2 & 13.3 & 1 & 10 & 1 & 100 \\
\hline Ugandal & 2 & 12.5 & 2 & 13.3 & 1 & 10 & 0 & 0 \\
\hline Total & 16 & 100 & 15 & 100 & 10 & 100 & 1 & 100 \\
\hline
\end{tabular}

Table 2: Lines of strains Mycobacterium tuberculosis and their octal code.

\begin{tabular}{|c|c|c|}
\hline $\mathbf{N}^{\circ}$ strains & Lines & Octal code \\
\hline 1 & LAM & 776177607760771 \\
\hline 2 & West African1 & 776177607760771 \\
\hline 3 & LAM3 & 776177607760771 \\
\hline 4 & LAM3 & 776177607760771 \\
\hline 5 & LAM3 & 770777777777671 \\
\hline 6 & West African1 & 7777777774020771 \\
\hline 7 & Haarlem 3 & 777777774020771 \\
\hline 8 & Haarlem 3 & 776177607760771 \\
\hline 9 & LAM3 & 777777777760730 \\
\hline 10 & Ugandal & 770777777777777671 \\
\hline 11 & West African 1 & 776177607760771 \\
\hline 12 & West African 1 & 776177607760771 \\
\hline 13 & LAM3 & 770777777777671 \\
\hline 14 & LAM3 & 777777777760730 \\
\hline
\end{tabular}

\section{LAM: Latin-American-Mediterranean}

As seen in this table, of 16 resistant strains, 10 were found in men $(62.5 \%)$. The age group 0-19 years was the most concerned (50\%) and $93.7 \%$ of resistant strains were found in the TPM+. The LAM line ranked first (43.7\%) followed by West African (31.3\%) and Haarlem and Ugandal, each with $12.5 \%$.

\section{Discussion}

The purpose of this study was to determine the prevalence of drug-resistant $\mathrm{TB}$ in family contacts and the different lines of circulating Mycobacterium tuberculosis strains. The present investigation revealed a frequency of $12.1 \%$ of isolated Mycobacterium strains [10] including 6 Africanum out of the 240 
samples analyzed. Sixteen strains (55.2\%) were resistant to at least one antituberculous drug. 15 strains (6.3\%) were MR-TB and among them, $4.2 \%$ preXDR [9] and 0.4\% XDR-TB [1]. Several different spoligotype profiles were identified among the genotyped isolates, indicating significant heterogeneity of the studied population. However, after phylogenetic analysis, many genotypes were attributed to only two main lineages, namely LAM $(43.7 \%)$ and West African (31.3\%).

The survey found a low preponderance of male sex (53.8\%), although not statistically significant, even considering drugresistant strains $(\mathrm{p}=0.46)$. Nevertheless, a study in Vietnam found that working-age male patients should be better controlled to prevent the emergence of drug-resistant TB [9]. Our observations are in line with some authors who show that the difference is due to the under-reporting of cases in women in underdeveloped countries. The authors showed that men accept testing more easily than women because of certain socio-cultural barriers $[11,12]$.

The high proportion of MDR-TB (46.7\%) found in the 0-19 age group and the pre and extensively resistant (70\% and 100\%) in the 20-54 age group is in line with many WHO reports. Several authors have also established an identical finding by showing that tuberculosis mainly affects the most mobile and economically active social strata [3-5]. These observations are consistent with that of a Vietnamese team led by Mai et al., Who reported that tuberculosis / HIV co-infection was associated with high rates of resistance to anti-TB drugs [9]. A similar trend has been reported by Eldholm, et al. [13] in a study on the impact of HIV co-infection on the development and transmission of MDR-TB. They have shown that HIV is an enhancer of TB outbreaks [13].

The prevalence of resistance reflects the quality of TB control in a community. The present study revealed a high proportion of resistance $(55.2 \%)$ in familial contacts of tuberculosis patients with the presence of MDR-TB (6.3\%) strains, pre- and ultra-resistant strains $(4.2 \%$ and $0,4 \%)$. The presence of drug-resistant strains in this work reflects poor TB management and poses a threat to health security. In addition, the survey also reports that $93.7 \%$ of drugresistant cases, including $93.3 \%$ of MDR-TB; $100 \%$ preXDR and $100 \%$ XDR-TB are TPM + cases that are responsible for the spread of drug-resistant strains in the population [3-5].

Indeed, the control of tuberculosis faces the problem of resistance to antituberculosis drugs. Patients with MDR-TB may remain contagious for a prolonged period and may be responsible for epidemics. This shows a real problem of patient care with impact on morbidity and mortality. There is therefore a need to strengthen surveillance, treatment and management of drug-resistant TB in the city as drug resistance is a major problem that creates barriers to TB treatment and control. Comparing the proportion found in the present study with the study of Kabedi, et al. in 2018, on the primary resistance to anti-tuberculosis drugs in PLWHA, we found that the primary resistance trend is however towards stabilization (54.3\% vs. 55.2\%) [5]. The short time difference between these two investigations probably explains this observation.

On the other hand, this proportion is higher than those of 3.5\%; $11 \% ; 24.7 \% ; 15.3 \%$ and $11.6 \%$ respectively obtained by Umubyeyi, et al., Catho, et al.; Perdigao, et al.; Hamusse, et al. and Fatemeh, et al. [14-18]. The differences in trend may be explained by differences in subject selection and the methodology applied in various studies. With respect to the typed of strains, the lineage classification based on the main genetic groups revealed that $43.7 \%$ of the strains [19] belonged to the LAM line. This Latin American and Mediterranean super-family (MAMA) is defined by the simultaneous absence of spacers 21-24 and 33-36 [20].

This result is in line with that obtained in children in Mexico, which shows that LAM strains were more represented (27.8\%) than the others [21]. In comparison with other African countries, the prevalence of LAM genotype in this study is lower than that of Angola (64.8\% Perdigao), but higher than that of Tanzania (3.8\% Kibiki) and Botwana (33\% Mogashoa) [16,22,23]. Considering other countries outside Africa, we note that the proportion of $43.7 \%$ is like those of $40.6 \%$ and $43.6 \%$ found respectively in Chile and Syria $[24,25]$. Generally, this LAM family is the largest in Venezuela (65\%), in the Mediterranean basin (for example, 34\% in Algeria, $55 \%$ in Morocco, 30\% in Spain) and in the Caribbean region (30\%) $[25,26]$.

The observations reported in this paper can be explained by the geographical location of DRC in the heart of Africa and the great tendency of populations to immigrate to other African countries and continents. The West African lineage is the most common in West Africa $[27,28]$, but currently, it is also found in other parts of the world, facilitated by migration of the population. The strains of Mycobacterium tuberculosis being strict human pathogens are therefore intimately associated with the migratory movements of human populations $[29,30]$. Our results showed a high frequency (31.3\%) compared to $1.25 \%$ and $17.4 \%$ found respectively by Taher, et al (Iran) and Jagielski, et al (Poland) [31,32].

About the Haarlem line, which is characterized by the absence of the 26-31 and 33-36 spacers, the survey reports a frequency of $12.5 \%$ [2] of which one preXDR and the other XDR. These strains are so called because found in people from Haarlem in the Netherlands [33-35]. Our data are consistent with those obtained by Sharereh (China), et al. in Teheran (Iran) in a work on resistance surveillance [33]. They showed that M. tuberculosis MDR, pre-XDR and XDR strains mainly belong to the Haarlem genotype $(11 / 23$, $47.8 \%$ ) [33-35].

Some authors have also shown that this line has an affinity with drug resistance $[3,4]$. This is the case of Ramazanzadeh, et al. and Jagielski et al. who showed in their investigations that the Haarlem 
line was associated with the drug resistance of Mycobacterium tuberculosis strains [32,34]. Haarlem is found in Europe, Central America and the Caribbean in the proportion of 25\% [36]. This suggests a link between Haarlem and European colonization [37]. Currently, its distribution is extensive in different geographical regions of the world such as Asia and Africa and has been documented [37].

However, this line of European origin was rarely found in India [37], which is not currently the case and it is thought that the migration of the population would be responsible for the spread of the strains on terrestrial globe. But we note that the geographical distribution of the strains is variable in the different regions [37]. Moreover, studies conducted in Iran (Middle East), show that this family represents more than half (57.1\%) of all strains grouped among Iranian patients with multidrug-resistant tuberculosis [37], thus requiring surveillance epidemiological, because they could cause serious epidemics. This shows that genotypic lineages are geographically variable.

The Ugandal line, which is very common in Uganda (70\%), is defined by the deletion of RD724 in combination with the lack of 33-36 spacers and spacer 40 and/or 43 by spoligotyping [38,39]. The present study reported a proportion of $12.5 \%$ found in MDRTB subjects. This frequency is lower than that of $46 \%$ obtained by Wamala in patients with tuberculous lymphadenitis in Uganda [39]. The presence of this lineage in the DRC can be explained by the displacement of the population. The very virulent Beijing genotype is a strain found mostly in Asia. It is significantly associated with drug resistance and incriminated in the spread and emergence of MDR-TB worldwide $[36,37,40,41]$.

Research in Central Asia has shown that the Beijing genotype is associated with increased resistance to antituberculosis drugs (rifampicin, Isoniazid and streptomycin) and is also involved in MDR-TB cases [41]. In 2006, Nikolayevskyy, et al. found similar results by reporting that the Beijing genotype was closely related to rifampicin and Isoniazid resistance and multidrug resistance in Ukraine [37]. Duong et al. showed that gyrA mutations were more common in the Beijing type, resulting in higher levels of resistance to Ofloxacin (OFX) [37]. This strain is also found in Africa as the Kibiki, et al. reported a proportion, though low, of 5.4\% in Tanzania [22]. However, this investigation did not reveal the presence of this line.

But given the DRC's closeness with other neighboring countries, which favors migratory movements, this lineage cannot be missed here. This requires more extensive studies to allow a good mapping of lineages in the city of Kinshasa and in different provinces of the country. The results of this study must consider certain limitations. The first concerns the weakness of typed strains that is attributable to the number of strains isolated during this study. This small number of strains does not make it possible to extrapolate the results.
The second is related to the spoligotyping technique used to type the strains, which is less discriminating compared to the MIRU VNTR technique, which is a more discriminating genotypic test to differentiate evolutionary potential strains within large families. However, these weaknesses do not fully affect the strength of the observations in this survey whose data may lead to broader and more comprehensive surveys.

\section{Conclusion}

Resistance is very high among family contacts. Drug-resistant strains were predominant in co-infected TB/HIV contacts in the DRC. The survey also revealed a genotypic lineage diversity of strains of Mycobacterium tuberculosis in Kinshasa. A more inclusive study of the Congolese population is needed to more accurately identify most of the dominant families in the country.

\section{Conflict of Interest}

None declared

\section{Acknowledgement}

I thank Mr. Mourad Gumusboga from the Institute of Tropical Medicine in Antwerp for agreeing to help us analyze strains by Spoligotyping.

\section{References}

1. World Health Organization (2019) Global tuberculosis control.

2. World Health Organisation (2015) Tuberculose: lignes directrices relatives à la surveillance de la pharmacorésistance. Cinquième édition, Geneva, Switzerland, p. 86.

3. Masjedi MR, Farnia P, Sorooch S, Pooramiri MV, Mansoori SD, et al. (2006) Extensively drug-resistant tuberculosis: 2 years of surveillance in Iran. Clin Infect Dis 43(7): 841-847.

4. Pillay M, Sturm AW (2007) Evolution of the extensively drug-resistant F15/LAM4/KZN strain of Mycobacterium tuberculosis in KwaZuluNatal, South Africa. Clin Infect Dis 45(11): 1409-1414.

5. Bajani MJK, Tumba JMK, Kalambay PK, Kalulu T, Kayumba, et al. (2019) Primary resistance of Mycobacterium tuberculosis to anti-Tuberculosis drugs in Person Living with HIV (PLHIV) in Kinshasa/DRC 5(2): $4027-$ 4031 .

6. Canetti G, Froman S, Grosset J, Hauduroy P, Langerova M, et al. (1963) Mycobacteria: laboratory methods for testing drug sensitivity and resistance. Bull World Health Organ 29(5): 565-578.

7. Groenen PMA, Bunschoten AE, van Soolingen D, van Embden JDA (1993) Nature of DNA polymorphism in the direct repeat cluster of Mycobacterium tuberculosis; Application for strain differentiation by a novel method. Mol. Microbiol 10(5): 1057-1065.

8. Mai TQ, Van Anh NT, Hien NT, Lan NH, Giang DC, et al. (2017) Drug resistance and Mycobacterium tuberculosis strain diversity in TB/HIV co-infected patients in Ho Chi Minh city, Vietnam. J Glob Antimicrob Resist 10: pp154-160.

9. Hirsh AE, Tsolaki AG, DeRiemer K, Feldman MW, Small PM (2004) Stable association between strains of Mycobacterium tuberculosis and their human host populations. Proc Natl Acad Sci USA 101: 4871-4876.

10. Azimi T, Nasiri MJ, Zamani S, Hashemi A, Goudarzi H, et al. (2018) High genetic diversity among Mycobacterium tuberculosis strains in Tehran, Iran. Journal of Clinical Tuberculosis and Other Mycobacterial Diseases 11: $1-6$ 
11. Borgdorff MW, Nagelkerke NJD, Dye C, Nunn P (2000) Genre et tuberculose: une comparaison entre les enquêtes de prévalence et les données de déclaration pour explorer les différences entre sexes dans la détection des cas. Int J Tuberc Lung Dis 4(2): 123-132.

12. Crampin AC, Glynn JR, Floyd S, Malema SS, Mwinnuka VK, et al. (2004) Tuberculose en fonction du sexe : exploration des types de la maladie dans une étude cas-contrôle au Malawi. Int J Tuberc Lung Dis 8(2): 194 203.

13. Eldholm V, Rieux A, Monteserin J, Lopez JM, Palmero D, et al. (2016) Impact of HIV co infection on the evolution and transmission of multidrug resistant tuberculosis. Elife 5

14. Umubyeyi AN, Rigouts L, Zissis G, Kamanzi E, Pauwels P, et al. (2007) Résistance primaire et acquise aux antituberculeux des souches de Mycobacterium tuberculosis isolées au Rwanda. Med Trop 67: 149-153.

15. Catho G, Senechal A, Ronnaux-Baron AS, Valour F, Perpoint T, et al. (2015) Children exposed to multidrug-resistant tuberculosis: How should we manage: Analysis of 46 child contacts and review of the literature. Rev PneumolClin 71(6): 335-341.

16. Perdigão J, Clemente S, Ramos J, Masakidi P, Machado D, et al. (2016) Genetic diversity, transmission dynamics, and drug resistance of Mycobacterium tuberculosis in Luanda, Angola. Int J Mycobacteriol 5(1): 38-39.

17. Hamusse SD, Teshome D, Hussen MS, Demissie M, Lindtjørn B (2016) Primary and secondary anti-tuberculosis drug resistance in Hitossa District of Arsi Zone, Oromia Regional State, Central Ethiopia. BMC Public Health 16: 593.

18. Mehravar F, Bazrafshan B, Abbasi A, Golsha R (2016) Primary MultidrugResistant Mycobacterium Tuberculosis (P-MDRTB) in Gorgan, Iran, IJHS 2(3): 6-9.

19. Goyal M, Saunders NA, van Embden JDA, Young DB, Shaw RJ (1997) Differentiation of Mycobacterium tuberculosis isolates by Spoligotyping and IS6110 restriction fragment length polymorphism. J Clin Microbiol 35: $647-651$.

20. Programme national de lutte contre la Tuberculose (2016) Programme antituberculeux intégré aux soins de santé de base $5^{\text {th }}$ édition (PATI 5). PNT/ RDC.

21. Sola C, Filliol I, Guterrez MC, Mokrousov I, Vincent V, (2001) Spoligotype database of Mycobacterium tuberculosis: biogeographic distribution of shared types and epidemiologic and phylogenetic perspectives, Emerg Infect Dis 7(3): 390-396.

22. Parra MM, Rodríguez JK, García JLA, López-Vidal Y, Castañón-Arreola M, et al. (2011) Mycobacterium tuberculosis Complex Genotype Diversity and Drug Resistance Profiles in a Pediatric Population in Mexico. Tuberc Res Treat pp. 239042.

23. Kibiki GS, Mulder B, Dolmans WM, de Beer JL, Boeree M, et al. (2007) M. tuberculosis genotypic diversity and drug susceptibility pattern in HIV-infected and non-HIV-infected patients in northern Tanzania. BMC Microbiol 7: 51.

24. Mogashoa T, Melamu P, Ley SD, Streicher EM, Iketleng T, et al. (2019) Genetic diversity of Mycobacterium tuberculosis strains circulating in Botswana. PLoS One 14(5): e0216306.

25. Lagos J, Couvin D, Arata L, Tognarelli J, Aguayo C, et al. (2016) Analysis of Mycobacterium tuberculosis Genotypic Lineage Distribution in Chile and Neighboring Countries. PLoS ONE 11(8): e0160434.

26. Bedrossian N, Rahmo A, Karam W, Hamze M (2013) Mycobacterium tuberculosis spoligotypes circulating in the Syrian population: A retrospective study. Int J Mycobacteriol 2: 141-147.
27. Duchêne V, Ferdinand, Filliol I, Guégan JF, Rastogi N, et al. (2004) Phylogenetic reconstruction of Mycobacterium tuberculosis within four settings of the Caribbean region: tree comparative analyses and first appraisal on their phylogeography. Infect Genet Evol 4(1): 5-14.

28. De Jong BC, Antonio M, Gagneux S (2010) Mycobacterium africanumreview of an important cause of human tuberculosis in West Africa. PLoS Negl Trop Dis 4(9): 744

29. Brudey K, Driscoll JR, Rigouts L, Prodinger WM, Gori A, et al. (2006) Mycobacterium tuberculosis complex genetic diversity: mining the fourth international spoligotyping database (SpolDB4) for classification, population genetics and epidemiology. BMC Microbiol 6: 23.

30. Gagneux S, DeRiemer K, Van T, Kato-Maeda M, de Jong BC, et al. (2006) Variable host-pathogen compatibility in Mycobacterium tuberculosis. Proc Natl Acad Sci USA 103: 2869-2873.

31. Jagielski T, Augustynowicz-Kopec E, Zozio T, Rastogi N, Zwolska Z (2010) Spoligotype-based comparative population structure analysis of multidrug-resistant and isoniazid-monoresistant Mycobacterium tuberculosis complex clinical isolates in Poland. J Clin Microbiol 48(11): 3899-3909.

32. Khanipour S, Ebrahimzadeh N, Masoumi M, Sakhaei F, Alinezhad F (2016) Haarlem 3 is the predominant genotype family in multidrugresistant and extensively drug-resistant Mycobacterium tuberculosis in the capital of Iran: A 5-year survey. Journal of Global Antimicrobial Resistance 5: 7-10.

33. Ramazanzadeh R, Roshani D, Shakib P, Rouhi S (2015) Prevalence and occurrence rate of Mycobacterium tuberculosis Haarlem family multidrug resistant in the worldwide population: A systematic review and meta-analysis. J Res Med Sci 20(1): 78-88.

34. Sola c, Rastogi N (2006) Molecular epidemiology and population genetics of tuberculosis. In: Ngeow YF, Yap SF (Eds.). Academy of Sciences of Malaysia, Kuala Lumpur pp. 23-68.

35. Nikolayevskyy V, Gopaul K, Balabanova Y, Brown T, Fedorin I, et al. (2006) Differentiation of tuberculosis strains in a population with mainly Beijing-family strains. Emerg Infect Dis 12: pp1406-1413.

36. Duong DA, Nguyen TH, Nguyen TN, Dai VH, Dang TM, et al. (2009) Beijing genotype of Mycobacterium tuberculosis is significantly associated with high-level fluoroquinolone resistance in Vietnam. Antimicrob Agents Chemother 53(11): 4835-4839.

37. Asiimwe BB, Koivula T, Kallenius G, Huard RC, Ghebremichael S, et al. (2008) Mycobacterium tuberculosis Uganda genotype is the predominant cause of TB in Kampala, Uganda. Int J Tuberc Lung Dis Off J Int Union Against Tuberc Lung Dis 12(4): 386-391.

38. Wamala D, Asiimwe B, Kigozi E, Mboowa G, Joloba M, et al. (2014) Clinicopathological features of tuberculosis due to Mycobacterium tuberculosis Uganda genotype in patients with tuberculous lymphadenitis: a cross sectional study. BMC Clin Pathol 14(1): 1472-6890.

39. Barnes PF, Cave MD (2003) Molecular epidemiology of tuberculosis. N Engl J Med 349: 1149-1156.

40. Gori A, Bandera A, Marchetti G, Degli Esposti A, Catozzi L, et al. (2005) Spoligotyping and Mycobacterium tuberculosis. Emerg Infect Dis 11(8): 1242-1248.

41. Cox HS, Kubica T, Doshetov D, Kebede Y, Rusch-Gerdess S, et al. (2005) The Beijing genotype and drug resistant tuberculosis in the Aral Sea region of Central Asia. Respir Res 6: pp134. 